Bundesgesundheitsbl 2019 - 62:1276-1277 https://doi.org/10.1007/s00103-019-03014-9 Online publiziert: 2 . September 2019 (c) Springer-Verlag GmbH Deutschland, ein Teil von Springer Nature 2019

\author{
Iris Pigeot ${ }^{1,2} \cdot$ Ronja Foraita $^{1} \cdot$ Cornelia Frömke ${ }^{3} \cdot$ Bernhard Haller ${ }^{4}$. \\ Verena Sophia Hoffmann ${ }^{5}$. Antonia Zapf \\ 'Leibniz-Institut für Präventionsforschung und Epidemiologie - BIPS, Bremen, Deutschland \\ ${ }^{2}$ Fachbereich 03: Mathematik und Informatik, Universität Bremen, Bremen, Deutschland \\ ${ }^{3}$ Fakultät III, Abteilung Information und Kommunikation, Hochschule Hannover, Hannover, Deutschland \\ ${ }^{4}$ Institut für Medizinische Informatik, Statistik und Epidemiologie; Klinikum rechts der Isar, Technische \\ Universität München, München, Deutschland \\ ${ }^{5}$ Institut für Diabetesforschung, Helmholtz Zentrum München, Deutsches Forschungszentrum für \\ Gesundheit und Umwelt, München-Neuherberg, Deutschland \\ ${ }^{6}$ Institut für Medizinische Biometrie und Epidemiologie, Universitätsklinikum Hamburg-Eppendorf, \\ Hamburg, Deutschland
}

\title{
Erratum zu: Ethische Bewertung von Studien am Menschen außerhalb des regulatorischen Rahmens: nicht bindend, aber von großer Wichtigkeit
}

\section{Erratum zu:}

Bundesgesundheitsbl 2019

https://doi.org/10.1007/s00103-019-

02947-5

In der Originalfassung des Artikels finden sich Bezüge auf Rechtsverordnungen, die während des Reviewprozesses außer Kraft gesetzt wurden, sowie zwei sprachliche Inkorrektheiten.

Im Abschnitt „Freie Studien und ihre Abgrenzung“ (S. 723) wurden Quellen zitiert, die bei Drucklegung nicht mehr gültig waren. Der folgende Absatz mit deren Literaturangaben wird wie folgt korrigiert und um die nun gültigen Quellen ergänzt:

Des Weiteren bedarf es gemäß der Strahlenschutzverordnung 2001 (StrlSchV; [10]) und der Röntgenverordnung (RöV; [11]) einer Stellungnahme der Ethikkommission im Zuge des Genehmigungsverfahrens beim Bundesamt für Strahlenschutz. Daher lassen sich die freien Studien auch am besten dadurch abgrenzen, dass sie weder unter den Begriff der klinischen Prüfung nach $\$ 4$ Abs. 23 Satz 1 AMG noch in den Geltungsbereich der $\$ \$ 20-23$ a MPG oder der $\$ \$ 23,24$ Abs. 1 Nr. 8, 92 StrlSchV 2001 bzw. der $\$ \$ 28 \mathrm{a}, 28$ b Abs. 1 Nr. 8, $28 \mathrm{~g}$ RöV fallen. Zum 31.12.2018 trat die Neufassung der StrlSchV (StrlSchV 2018; [1]) in Kraft. Seitdem sind für Verfahren zur Anwendung radioaktiver Stoffe oder ionisierender Strahlung am Menschen zum Zweck der medizinischen Forschung das Strahlenschutzgesetz (StrlSchG, [2]) und die StrlSchV 2018 einschlägig.

Die Literaturstellen [10] und [11] in der Originalpublikation wurden überarbeitet und sind unten als [3] und [4] als Ergänzung aufgeführt.

Im Abschnitt „Interventionsstudien“ (S. 727) wird das Vorgehen bei der Einholung der Einverständniserklärung zur Teilnahme von Kindern in der IDEFICSStudie (Laufzeit: 09/2006-02/2012) zur Verdeutlichung wie folgt korrigiert und ergänzt:

Die Einwilligungserklärung für das Kind musste von beiden Elternteilen (Sorgeberechtigten) unterschrieben werden. Zusätzlich wurde jedes Kind vor

Die Online-Version des Originalartikels ist unter https://doi.org/10.1007/s00103-019-02947-5 zufinden. 
jedem Untersuchungselement nach kindgerechter Aufklärung [5] um mündliche Zustimmung gebeten.

Siehe auch die Literaturstellen $[25,26]$ in der Originalarbeit.

In der „Diskussion“ (S. 727) steht: Für klinische Ethikkommissionen wird daher nach dem AMG die Beteiligung ,einer Person mit Erfahrung auf dem Gebiet der Versuchsplanung und Statistik" gefordert.

Richtig heißt es: Für medizinische Ethikkommissionen wird daher nach dem AMG die Beteiligung „einer Person mit Erfahrung auf dem Gebiet der Versuchsplanung und Statistik" gefordert.

\section{Korrespondenzadresse}

\section{Ronja Foraita}

Leibniz-Institut für Präventionsforschung und Epidemiologie - BIPS

Achterstr. 30, 28359 Bremen, Deutschland

foraita@leibniz-bips.de

\section{Literatur}

1. Bundesministerium der Justiz für Verbraucherschutz (2018) Verordnung über den Schutz vor Schäden durch ionisierende Strahlen (Strahlenschutzverordnung - StrlSchV). http://www. gesetze-im-internet.de/strlschv_2018/StrlSchV. pdf. Zugegriffen: 25. Juli 2019

2. Bundesministerium der Justiz für Verbraucherschutz (2017) Gesetz zum Schutz vor der schädlichen Wirkung ionisierender Strahlung (Strahlenschutzgesetz - StrlSchG). http://www. gesetze-im-internet.de/strlschg/StrlSchG.pdf. Zugegriffen:25. Juli 2019

3. Bundesministerium der Justiz für Verbraucherschutz (2001) Verordnung über den Schutz vor Schäden durch ionisierende Strahlen (Strahlenschutzverordnung - StrlSchV). https://www. buzer.de/gesetz/871/index.htm. Zugegriffen: 25 . Juli 2019 ([Außerkraftsetzung am 31.12.2018])

4. Bundesministerium der Justiz für Verbraucherschutz (2014) Verordnung über den Schutz vor Schäden durch Röntgenstrahlen (Röntgenverordnung - RöV). https://www.buzer.de/gesetz/4307/ index.htm. Zugegriffen:31. Juli 2019 ([Außerkraftsetzung am 31.12.2018])

5. Ahrens W, Bammann K, Siani A et al (2011) The IDEFICS cohort: design, characteristics and participation in the baseline survey. Int J Obes 35(Suppl 1):S3-S15 